\title{
ATUAÇÃO DAS UNIVERSIDADES PROMOTORAS DE SAÚDE NA PREVENÇÃO DE NEOPLASIAS DO COLO DO ÚTERO
}

\section{Karen Fernanda de Deus Dantas}

Discente de medicina Centro Universitário de Maringá - UNICESUMAR, Maringá, PR, Brasil.

\section{Tamara Fadoni Sahyun Abdalla}

Discente de medicina Centro Universitário de Maringá - UNICESUMAR, Maringá, PR, Brasil.

\section{Mirian Ueda Yamaguchi}

Farmacêutica. Doutora e Docente no Programa de Pós-graduação Stricto sensu em Promoção da Saúde - PPGPS do Centro Universitário de Maringá UNICESUMAR. Pesquisadora do Instituto Cesumar de Ciência, Tecnologia e Inovação - ICETI, Brasil.

\section{Tânia Maria Gomes Silva}

Doutora em História, pela Universidade Federal do Paraná - UFPR. Docente no Pós-graduação Stricto sensu em Promoção da Saúde - PPGPS do Centro Universitário de Maringá - UNICESUMAR Pesquisadora do Instituto Cesumar de Ciência Tecnologia e Inovação - ICETI, Maringá, PR, Brasil.

\section{Marcelo Picinin Bernuci}

Docente no Programa de Pós-graduação Stricto sensu em Promoção da Saúde - PPGPS do Centro Universitário de Maringá - UNICESUMAR. Pesquisador do Instituto Cesumar de Ciência, Tecnologia e Inovação - ICETI, Brasil.
Autor correspondente

Marcelo Picinin Bernuci

marcelo.bernuci@unicesumar.edu.br
RESUMO: Estudo descritivo de corte transversal realizado com mulheres $(n=61)$ que atuam no setor de limpeza de uma instituição de ensino superior privada no município de Maringá-PR. O objetivo foi avaliar a percepção delas sobre a prevenção da neoplasia do colo do útero. Foram aplicados questionários semiestruturados, elaborados com base nas informações do Instituto Nacional do Câncer. Verificouse que $8,20 \%$ das funcionárias nunca realizaram o preventivo (grupo NR); $26,23 \%$ realizaram há mais de dois anos (grupo $\mathrm{R}+2$ ); $65,57 \%$ há menos de dois anos (grupo R-2). Para NR, 20\% reconhecem o HPV como fator necessário para o câncer, $43,75 \%$ para NR, e $40 \%$ para $\mathrm{R}+2$. Para NR, 60\% reconhecem que o preventivo auxilia no diagnóstico, $70 \%$ para $\mathrm{R}+2$, e $80 \%$ para $\mathrm{R}-2$. Para $\mathrm{R}+2,25 \%$ reconhecem que toda mulher entre 25 e 64 anos de idade deve fazer o exame, e 7,5\% para R-2. Para $\mathrm{R}+2,12,5 \%$ têm hábito de realizar exames rotineiramente, $\mathrm{e}$ $30 \%$ para R-2. Conclui-se que a adesão ao exame preventivo é intuitiva e o conhecimento sobre a doença é limitado, ressaltando a necessidade de educação em saúde.

PALAVRAS-CHAVE: Promoção da saúde; Educação em saúde; Neoplasias do colo do útero.

\section{HEALTH-PROMOTING UNIVERSITIES IN THE PREVENTION OF CERVIX CANCER}

ABSTRACT: Current transversal and descriptive study was undertaken with females $(n=61)$ who work in the cleaning sector of a private university in Maringá, Brazil. Their perception on the prevention of cervix cancer was evaluated. Half-structured questionnaires, prepared on information retrieved from database of the Cancer National Institute, were applied. Further, $8.20 \%$ of the employees never undertook any preventive examination (group NR); $26.23 \%$ undertook the test more than two years ago (group $\mathrm{R}+2$ ); $65.57 \%$ undertook the test less than two years ago (group R-2). For NR, 20\% acknowledge HPV as a necessary factor for cancer; $43.75 \%$ for NR and $40 \%$ for $\mathrm{R}+2$. In the case of NR, $60 \%$ acknowledge that prevention helps in diagnosis, $70 \%$ in $\mathrm{R}+2$ and $80 \%$ in $\mathrm{R}-2$. In the case of $\mathrm{R}+2,25 \%$ acknowledge that every female between 25 and 64 years should undertake the test and $7.5 \%$ in the case of $\mathrm{R}-2$. In the case of $\mathrm{R}+2,12.5 \%$ have routine tests and 30\% for R-2. Results show that adhesion to preventive test is intuitive and knowledge on the disease is limited. Health education is highly recommended.

KEYWORDS: Health Promotion; Health Education; Uterine Cervical Neoplasms. 


\section{INTRODUÇão}

A neoplasia do colo do útero ocorre em todo o mundo, mas as maiores taxas de incidência estão nos países em desenvolvimento ${ }^{1}$. No Brasil, estimamse 16.370 novos casos para o biênio 2018-2019, com variações regionais, tendo a região Sul, a quarta posição de prevalência ${ }^{2}$. Este tipo de neoplasia caracteriza-se pela replicação desordenada do epitélio de revestimento do útero e possui uma evolução lenta, passando pelas fases pré-clínicas, que são detectáveis e curáveis ${ }^{3}$. O fator necessário para a ocorrência dessa neoplasia é a infecção primária pelo vírus HPV (Papilomavírus humano), mas há outros fatores de risco, como imunidade, genética, atividade sexual precoce, multiplicidade de parceiros, uso de contraceptivos orais e tabagismo ${ }^{4}$.

O método amplamente utilizado para detecção das lesões precursoras da neoplasia do colo do útero é o teste de Papanicolau. O rastreamento no Brasil é oportunístico, isto é, não há organização prévia para atingir a população-alvo ${ }^{5}$, diferentemente do que ocorre nos países desenvolvidos ${ }^{6}$. As etapas do rastreio seguem as diretrizes do Ministério da Saúde, implicando na identificação e convite às mulheres, garantia dos recursos humanos e materiais, disponibilização de exames de qualidade, assegurando o tratamento e cuidado com aquelas que apresentarem alterações nos exames5. Propõe-se o rastreamento às mulheres com vida sexual ativa, assintomáticas e com idade entre 25 e 64 anos, a cada três anos, após dois exames anuais consecutivos normais 7 . A cobertura de, no mínimo, $80 \%$ do públicoalvo no exame citopatológico, somada ao diagnóstico e tratamento adequado, viabilizaria a redução de 60 a 90\% dos casos de neoplasias do colo do úteró ${ }^{4}$.

No Brasil, há dificuldade para o controle de mulheres que não estão realizando o rastreio, dada a indisponibilidade de um sistema de informação de base populacional identificatório daquelas que realizam o procedimento profilático e das que não o realizam ${ }^{7}$. Consequentemente, há mulheres rastreadas além do necessário e outras que nunca passam por tal procedimento. Dentre as barreiras para a adesão ao rastreio destacam-se o medo do resultado dos exames, a falta de informação, a dificuldade de acesso à
Unidade Básica de Saúde (UBS), a falta de vínculo com o profissional da saúde ${ }^{8}$, desconhecimento do próprio corpo, falta de oportunidade para a usuária do serviço de saúde falar sobre si e sobre sua sexualidade, acrescido de medo, vergonha, tabus e ideias preconceituosas sobre a mulher'.

O estudo aqui apresentado se insere nas medidas preventivas que têm como propósito contribuir para o empoderamento em saúde, na busca de melhores níveis de saúde, qualidade de vida e equidade, notadamente por meio da autonomia dos indivíduos e das comunidades ${ }^{10}$. Considerando-se que o tratamento tardio eleva as taxas de mortalidade da neoplasia do colo do útero, fomentar o diagnóstico precoce constitui-se numa estratégia de promoção da saúde ${ }^{11}$. Neste contexto, vale destacar a importância das Universidades Promotoras de Saúde enquanto espaços de formação de futuros profissionais e de gestores de políticas públicas na perspectiva da equidade, mediante o incentivo de envolvimento entre espaço acadêmico e comunidade ${ }^{12,13}$.

Inserido neste ambiente de uma Universidade Promotora de Saúde e buscando contribuir para o aprofundamento da discussão, o presente estudo tem como objetivo avaliar a percepção de funcionárias de uma instituição de ensino superior (IES) sobre a prevenção da neoplasia do colo do útero, bem como os motivos indutores para a realização do exame preventivo.

\section{MÉTODOS}

Trata-se de um estudo descritivo de corte transversal com abordagem quantitativa, realizado com mulheres que trabalham no serviço de limpeza de uma instituição de ensino superior (IES) privada, localizada no município de Maringá, região Norte do Estado do Paraná, no sul do Brasil. A opção pelo público-alvo levou em conta que baixa escolaridade e renda estão associados à não adesão ao exame citopatológico, com maiores riscos de desenvolvimento de neoplasia do colo uterino ${ }^{1}$.

Este estudo foi aprovado pelo Comitê de Ética em Pesquisa (CEP) em humanos do Centro Universitário Cesumar, sob o parecer número 2.529.524, seguindo a resolução 466/12 do Conselho Nacional de Saúde.

Os dados foram adquiridos por meio de 
entrevistas realizadas no próprio local de trabalho das funcionárias durante atividade em grupo promovida pela IES. Esta atividade baseia-se em reuniões de diálogo de segurança semanal (DSS) com todos os funcionários e funcionárias que ocupam serviço de risco da IES. Esses encontros ocorrem nos três turnos de trabalho: matutino, vespertino e noturno. $\mathrm{O}$ convite para que as mulheres participassem do presente estudo foi feito às integrantes de cada período.

A coleta de dados ocorreu em duas etapas. $\mathrm{Na}$ primeira etapa, convocou-se a população de funcionárias da IES $(n=87)$ a comparecer à reunião onde foram explicados os objetivos do estudo e aplicado o Termo de Consentimento Livre e Esclarecido (TCLE). Todas as mulheres aceitaram participar e assinaram o TCLE. Neste mesmo encontro, todas preencheram um questionário sociodemográfico, cujos dados foram analisados para determinação da amostra. Os critérios de elegibilidade adotados foram: a) ter idade entre 25 até 64 anos, período esse estabelecido, com base na preconização do Ministério da Saúde, para a realização do exame citopatológico ${ }^{14}$; b) falar, escrever e compreender o idioma português, considerando-se que na IES onde o estudo foi realizado há mulheres de origem haitiana que não dominam a língua portuguesa do Brasil. A análise desses dados subsidiou a seleção de 61 mulheres que se enquadraram nos critérios de elegibilidade e participaram da segunda etapa do estudo.

$\mathrm{Na}$ segunda etapa, as mulheres elegíveis foram convocadas para uma segunda reunião onde preencheram um segundo questionário semiestruturado a fim de identificar a percepção das mesmas quanto à prevenção da neoplasia do colo do útero. O questionário foi elaborado com base nas informações do $\mathrm{INCA}^{7}$ acerca da prevenção deste tipo de neoplasia e continha 36 perguntas. As perguntas buscavam abordar fatores de risco para o desenvolvimento da neoplasia do colo do útero, verificar o conhecimento acerca dos métodos preventivos, bem como avaliar autocuidado, comportamentos de prevenção e razões indutoras para a realização do exame preventivo. Por fim, uma questão aberta indagava sobre a preferência das mulheres quanto ao melhor meio para receber informações de saúde.

A análise destes questionários possibilitou a organização das mulheres em grupos, de acordo com o período de realização do exame citopatológico para rastreio das lesões sugestivas de malignidade. Os grupos foram divididos em: 1) mulheres que nunca realizaram o exame citopatológico - Grupo NR ( $n=5) ; 2)$ mulheres que realizaram o exame citopatológico no período maior ou igual a dois anos - Grupo $\mathrm{R}+2(\mathrm{n}=16)$; e 3$)$ mulheres que realizaram o exame citopatológico no período menor que dois anos - Grupo R-2 $(n=40)$.

Os dados foram descritos por meio de tabelas de frequências. As análises descritivas foram realizadas com auxílio do programa Statistical Analysis Software (SAS, version 9.0).

\section{RESULTADOS}

Das 61 participantes do estudo apenas 8,20\% declararam nunca ter realizado o exame preventivo para a neoplasia do colo do útero (Grupo NR), enquanto que $26,23 \%$ realizaram há mais de dois anos (Grupo $\mathrm{R}+2)$ e 65,57\% realizaram há menos de dois anos (Grupo R-2). Para o Grupo NR, a maioria declarou idade entre 30 a 39 anos (60\%); possuir ensino fundamental incompleto (40\%); ter renda familiar de até $\mathrm{R} \$ 1.760$ reais (40\%); possuir cônjuge (40\%); e filhos (60\%). Para o Grupo R+2, a maioria declarou idade entre 40 a 49 anos (43,75\%); possuir ensino médio completo (25\%); renda familiar de até $\mathrm{R} \$ 1.760,00$ (50\%); possuir cônjuge $(56,25 \%)$; e filhos (81,25\%). Para o Grupo R-2, a maioria declarou idade entre 30 a 39 anos (35\%); possuir ensino médio completo (42,5\%); renda familiar de até $\mathrm{R} \$ 1.760,00$ (52,5\%); possuir cônjuge $(57,5 \%)$; e filhos (80\%) (dados não mostrados).

Os dados referentes à caracterização dos fatores de risco para o desenvolvimento da neoplasia do colo do útero estão apresentados na Tabela 1 . Nota-se que a maioria das mulheres declarou ter iniciado a atividade sexual entre os 15 e 20 anos de idade, sendo $40 \%$ para NR; 68,75\% para R+2; e 75\% para R-2. A maioria também declarou ter tido apenas um parceiro sexual ao longo de um período de um ano, sendo 60\% para NR; $75 \%$ para $\mathrm{R}+2$; e $65 \%$ para R-2. Quanto aos métodos contraceptivos, $80 \%$ das mulheres do grupo NR declararam não fazer uso; enquanto 31,5\% para $\mathrm{R}+2$; e 32,5\% para $\mathrm{R}-2$. 
Tabela 1. Caracterização dos fatores de risco para o desenvolvimento da neoplasia do colo do útero

\begin{tabular}{|c|c|c|c|}
\hline & Grupo NR $(n=5)$ & Grupo $R+2(n=16)$ & Grupo R-2 $(n=40)$ \\
\hline \multicolumn{4}{|l|}{ Início da atividade sexual } \\
\hline Antes dos 15 & - & $6,25 \%(n=1)$ & $12.5 \%(n=5)$ \\
\hline $15-20$ & $40 \%(n=2)$ & $68,75 \%(n=11)$ & $75 \%(n=30)$ \\
\hline $21-25$ & - & $25 \%(n=4)$ & $7.5 \%(n=3)$ \\
\hline $26-30$ & - & - & $2.5 \%(\mathrm{n}=1)$ \\
\hline Depois dos 30 & $20 \%(n=1)$ & - & $2.5 \%(\mathrm{n}: 1)$ \\
\hline Não iniciou & $20 \%(n=1)$ & - & - \\
\hline Não respondido & $20 \%(n=1)$ & - & - \\
\hline \multicolumn{4}{|l|}{ Parceiros ao longo de 1 ano } \\
\hline 1 & $60 \%(n=3)$ & $75 \%(n=12)$ & $65 \%(n=26)$ \\
\hline 2 & - & $12,50 \%(n=2)$ & $15 \%(n=6)$ \\
\hline 3 & - & - & $7.5 \%(n=3)$ \\
\hline 4 & - & - & - \\
\hline 5 ou mais & - & $6,25 \%(n=1)$ & $5 \%(n=2)$ \\
\hline \multicolumn{4}{|l|}{ Contracepção } \\
\hline Camisinha & - & $25 \%(n *=4)$ & $17,5 \%(n=7)$ \\
\hline Anticoncepcional & - & $31,5 \%(n=5)$ & $32,5 \%\left(n^{*}=13\right)$ \\
\hline Injetável & - & $6,25 \%(\mathrm{n}=1)$ & $7,5 \%(n=3)$ \\
\hline Definitivos & - & $18,75 \%(\mathrm{n}=3)$ & $15 \%(n=6)$ \\
\hline Não fazem uso & $80 \%(n=4)$ & $31,5 \%(\mathrm{n}=5)$ & $32,5 \%\left(n^{*}=13\right)$ \\
\hline Método comportamental & - & - & $2,5 \%(\mathrm{n}=1)$ \\
\hline DIU & $20 \%(n=1)$ & - & $2,5 \%(\mathrm{n}=1)$ \\
\hline
\end{tabular}

Fonte: Dados da pesquisa.

A Tabela 2 apresenta os dados referentes à caracterização do conhecimento das mulheres sobre os métodos de rastreio e prevenção da neoplasia do colo do útero. Quanto à prevenção, a maioria declarou que o HPV não é um fator necessário, $80 \% \mathrm{NR} ; 50 \% \mathrm{R}+2$; $60 \%$ R-2. Ademais, a maioria das mulheres declarou que a transmissão do HPV é pela via vaginal, $40 \% \mathrm{NR}$; $81,25 \% \mathrm{R}+2 ; 80 \% \mathrm{R}-2$. Quanto aos fatores que ajudam a prevenir, $60 \%$ das mulheres do grupo NR declararam que é a vacina, enquanto que $87,5 \%$ e $90 \%$ dos grupos $\mathrm{R}+2$ e $\mathrm{R}-2$, respectivamente, responderam ser o rastreio por meio do exame preventivo. Quanto ao conhecimento da faixa etária para a vacina do HPV, a maioria indicou idades não preconizadas pelo Ministério da Saúde, $60 \%$ NR; $62,5 \% \mathrm{R}+2 ; 55 \% \mathrm{R}-2$. Quanto ao significado do diagnóstico precoce, a maioria identificou ser o exame preventivo, $60 \% \mathrm{NR} ; 75 \% \mathrm{R}+2 ; 80 \% \mathrm{R}-2$. Quanto à periodicidade para a realização do exame preventivo, a maioria $(60 \%, 56,25 \%)$ das mulheres do grupo NR e $\mathrm{R}+2$, respectivamente, indicou a cada um ano, se após dois exames consecutivos normais, com intervalo de seis meses, e anualmente para as do grupo R-2 (55\%). Em relação à faixa etária recomendada para realização do exame, a maioria declarou ter idade diferente de 25 a 64 anos, $60 \% \mathrm{NR} ; 75 \% \mathrm{R}+2 ; 90 \% \mathrm{R}-2$.

Tabela 2. Caracterização do conhecimento das mulheres sobre os métodos de rastreio e prevenção da neoplasia do colo do útero

(Continua)

\begin{tabular}{lccc}
\hline & Grupo NR (n=5) & Grupo R+2 (n= 16) & Grupo R-2 (n= 40) \\
\hline Fator necessário (HPV) & & & \\
\hline Sim & $20 \%(\mathrm{n}=2)$ & $43,75 \%(\mathrm{n}=7)$ & $40 \%(\mathrm{n}=16)$ \\
Não & $80 \%(\mathrm{n}=3)$ & $50 \%(\mathrm{n}=8)$ & $60 \%(\mathrm{n}=24)$ \\
$\quad$ Não responderam & - & $6,25 \%(\mathrm{n}=1)$ & - \\
\hline Forma de transmissão (HPV) & & & $80 \%(\mathrm{n}=32)$ \\
$\quad$ Via vaginal & $40 \%(\mathrm{n}=2)$ & $81,25 \%(\mathrm{n}=13)$ & $7,5 \%(\mathrm{n}=3)$ \\
Outras & $40 \%(\mathrm{n}=2)$ & $12,5 \%(\mathrm{n}=2)$ & $12,5 \%(\mathrm{n}=5)$ \\
$\quad$ Não responderam & $20 \%(\mathrm{n}=1)$ & $6,25 \%(\mathrm{n}=1)$ & \\
\hline Fatores que ajudam a prevenir & & & \\
\hline$\quad$ Vacina HPV & $60 \%(\mathrm{n}=3)$ & $56.25 \%(\mathrm{n} *=9)$ & $57.5 \%(\mathrm{n} *=23)$ \\
\end{tabular}


(Conclusão)

\begin{tabular}{|c|c|c|c|}
\hline & Grupo NR $(n=5)$ & Grupo $R+2(n=16)$ & Grupo R-2 $(n=40)$ \\
\hline \multicolumn{4}{|l|}{ Fatores que ajudam a prevenir } \\
\hline Uso de camisinha & $20 \%(n=1)$ & $56,25 \%(\mathrm{n} *=9)$ & $57.5 \%(n *=23)$ \\
\hline Rastreio por meio do exame preventivo & $40 \%(n=2)$ & $87,5 \%(n *=14)$ & $90 \%\left(n^{*}=36\right)$ \\
\hline Responderam outros & - & $12.5 \%(\mathrm{n}=2)$ & $27.5 \%(\mathrm{n}=11)$ \\
\hline \multicolumn{4}{|l|}{ Faixa etária (vacina) } \\
\hline Preconizada (MS) & $40 \%(n=2)$ & $37,5 \%(n=6)$ & $37,5 \%(n=15)$ \\
\hline Outras & $60 \%(n=3)$ & $62,5 \%(n=10)$ & $55 \%(\mathrm{n}=22)$ \\
\hline Não responderam & - & - & $7,5 \%(n=3)$ \\
\hline \multicolumn{4}{|l|}{ Diagnóstico precoce } \\
\hline Exame preventivo & $60 \%(n=3)$ & $75 \%(n=12)$ & $80 \%(n=32)$ \\
\hline Outros & . & $25 \%(n=4)$ & $15 \%(n=6)$ \\
\hline Não responderam & $40 \%(n=2)$ & - & $5 \%(n=2)$ \\
\hline \multicolumn{4}{|l|}{ Periodicidade para realização do exame } \\
\hline Anualmente & $20 \%(n=1)$ & $37,5 \%(n=6)$ & $55 \%(\mathrm{n}=22)$ \\
\hline $\begin{array}{l}\text { A cada } 1 \text { ano, se após } 2 \text { exames consecutivos normais, com } \\
\text { intervalo de } 6 \text { meses }\end{array}$ & $60 \%(n=3)$ & $56,25 \%(\mathrm{n}=9)$ & $32,5 \%(n=13)$ \\
\hline $\begin{array}{l}\text { A cada } 3 \text { anos, se após } 2 \text { exames consecutivos normais, com } \\
\text { intervalo de } 1 \text { ano }\end{array}$ & - & $6,25 \%(n=1)$ & $5 \%(\mathrm{n}=2)$ \\
\hline Não responderam & $20 \%(n=1)$ & - & $7,5 \%(n=3)$ \\
\hline \multicolumn{4}{|l|}{ Faixa etária recomendada para realização do exame } \\
\hline $25-64$ & $20 \%(n=1)$ & $25 \%(n=4)$ & $5 \%(n=2)$ \\
\hline Outras & $60 \%(n=3)$ & $75 \%(n=12)$ & $90 \%(n=36)$ \\
\hline Não responderam & $20 \%(n=1)$ & - & $5 \%(\mathrm{n}=2)$ \\
\hline
\end{tabular}

* = mais de uma alternativa foi assinalada pela mesma mulher.

Fonte: Dados da pesquisa.

Os dados referentes aos motivos que levaram as mulheres a realizarem o exame preventivo para o câncer do colo do útero estão apresentados na Tabela 3. Nota-se que, para mulheres do grupo $\mathrm{R}+2$, os principais motivos que as levaram a fazer o exame foram: entender ser importante fazer (18,75\%) e pela campanha (18,75\%). Já para aquelas do grupo R-2, os principais motivos foram porque entende ser importante fazer (45\%) e porque é um controle de problemas ou rotina (20\%).

Tabela 3. Caracterização dos motivos que levaram as mulheres à realização do exame preventivo para a neoplasia do colo do útero

\begin{tabular}{|c|c|c|}
\hline & Grupo $R+2(n=16)$ & Grupo R-2 $(n=40)$ \\
\hline O médico solicitou porque na minha família existe caso de CA & - & $2,5 \%(n=1)$ \\
\hline Tenho medo de desenvolver o CA do colo do útero & $12,50 \%(n=2)$ & $2,5 \%(n=1)$ \\
\hline Toda mulher entre 25 e 64 anos deve fazer & $25 \%(n=4)$ & $7,5 \%(n=3)$ \\
\hline Faço o exame para acompanhamento/controle de problemas ou rotina & $12,50 \%(n=2)$ & $30 \%(n=12)$ \\
\hline Porque acho importante fazer & $18,75 \%(n=3)$ & $45 \%(n=18)$ \\
\hline Porque teve campanha & $18,75 \%(n=3)$ & $2,5 \%(n=1)$ \\
\hline Por indicaçôes & $6,25 \%(n=1)$ & - \\
\hline Não responderam & $6,25 \%(n=1)$ & $10 \%(n=4)$ \\
\hline
\end{tabular}

Fonte: Dados da pesquisa. 
A Tabela 4 apresenta os dados referentes aos meios de preferência de receber informações sobre a prevenção da neoplasia do colo do útero pelas participantes do estudo. Nota-se que para aquelas do grupo NR, 60\% delas preferem ser informadas pelos médicos; já para os grupos $\mathrm{R}+2$ e R-2, a preferência da maioria foi a informação por palestras, $56,25 \%$ e $80 \%$, respectivamente.

Tabela 4. Caracterização dos meios de preferência de receber informações sobre a prevenção da neoplasia do colo do útero

\begin{tabular}{cccc}
\hline & Grupo NR $(\mathbf{n}=\mathbf{5})$ & Grupo R+2 $(\mathbf{n}=\mathbf{1 6})$ & Grupo R-2 $(\mathbf{n}=\mathbf{4 0 )}$ \\
\hline Palestras & $40 \%(\mathrm{n}=2)$ & $56,25 \%(\mathrm{n} *=9)$ & $80 \%(\mathrm{n} * 32)$ \\
Panfletos & - & $6,25 \%(\mathrm{n}=1)$ & $25 \%(\mathrm{n}=10)$ \\
Aula & - & $6,25 \%(\mathrm{n}=1)$ & $10 \%(\mathrm{n}=4)$ \\
Cartilhas & - & $6,25 \%(\mathrm{n}=1)$ & $15 \%(\mathrm{n}=6)$ \\
WhatsApp & $20 \%(\mathrm{n}=1)$ & $12,5 \%(\mathrm{n}=2)$ & $20 \%(\mathrm{n}=8)$ \\
Amigas & - & $12,5 \%(\mathrm{n}=2)$ & $10 \%(\mathrm{n}=4)$ \\
Médicos & $60 \%(\mathrm{n} * 3)$ & $50 \%(\mathrm{n}=8)$ & $55 \%(\mathrm{n}=22)$ \\
Outras & - & $6,25 \%(\mathrm{n}=1)$ & - \\
Não responderam & - & $6,25 \%(\mathrm{n}=1)$ & $10 \%(\mathrm{n}=4)$ \\
\hline
\end{tabular}

* = mais de uma alternativa foi assinalada pela mesma mulher.

Fonte: Dados da pesquisa.

\section{DISCUSSÃO}

A falta de informação e conhecimento demostram ser as principais barreiras encontradas pelas mulheres para ter acesso aos métodos de prevenção da neoplasia do colo do útero. Informação que pode ser válida, inclusive, para que se entenda o processo de adesão de outras neoplasias e subsidie eventuais campanhas.

A maioria das mulheres que nunca realizou o exame preventivo possui idade entre 30-39. Este fato gera preocupação, pois embora a média de idade em que as mulheres são diagnosticadas com câncer seja de 45 a 49 anos, as lesões de baixo/alto grau ou carcinoma in situ são comuns na faixa etária média de 36 anos $^{15}$. Além disso, essas mulheres relataram possuir grau de escolaridade inferior ao ensino fundamental, chamando também a atenção quanto à relação entre escolaridade e adesão ao rastreio para este tipo de câncer.

Há evidências de que entre as mulheres diagnosticadas com neoplasia do colo do útero, a maioria não possui ensino fundamental ${ }^{16}$. Há uma redução na probabilidade de lesões cervicais anormais em mulheres com maiores índices de alfabetização ${ }^{17}$, bem como se sabe que mulheres com baixo nível de escolaridade estão mais propensas a não comparecerem ao rastreio da neoplasia do colo do útero ${ }^{18}$. Reforça-se, portanto, a necessidade de um olhar mais atencioso para aquelas que possuem um nível educacional menor, pois esse aspecto influencia diretamente na capacidade de compreensão dos fatores de risco e a vulnerabilidade a que estão expostas.

Evidenciou-se que a maioria das mulheres está exposta aos mesmos fatores de risco, como início da atividade sexual precoce, não uso de camisinha e o uso de anticoncepcional oral. Acredita-se que essa exposição similar aos fatores de risco se deva ao fato de a maioria também ter cônjuge e, assim, por convicções e crenças, não fazer o uso de preservativo.

Estudos demonstram que o fato de ter um cônjuge diminui consideravelmente o uso de preservativos e isso se dá pelo fato de as mulheres confiarem em seus parceiros, dispensando, assim, o uso de preservativos ${ }^{19,20}$. Tal evidência, apesar de trazer riscos para as mulheres, configura a comum relação marital, na qual a confiança deve ser uma das bases. Nesse sentido, as políticas de saúde precisam estar atentas às questões culturais. Por outro lado, não se pode ignorar o fato de que esse fator de risco é modificável na população entrevistada, diferente dos demais, e exerce certa influência no desenvolvimento de neoplasias.

O hábito de não utilizar preservativos tem sido relatado por mulheres diagnosticadas com neoplasia do colo do útero ${ }^{21}$, exigindo medidas gerais de orientação da transmissão do vírus do HPV, como o incentivo ao uso de preservativos. Além do mais, observou-se que a 
maioria das mulheres desconhece o vírus do HPV como fator necessário para o desenvolvimento da neoplasia do colo do útero, reforçando a necessidade de discussões sobre o tema.

Foi evidenciado desconhecimento sobre a faixa etária indicada para a vacina contra o HPV ou para a realização do exame Papanicolau, assim como também acerca da periodicidade que o preventivo deve ser realizado. Há evidências de que quanto menor o nível de conhecimento sobre o HPV e a neoplasia do colo do útero menor o grau de consciência da gravidade da doença, e como consequência disso menos frequentes são as atitudes tomadas para prevenção ${ }^{22}$.

As mulheres que participaram do presente estudo reconhecem o exame preventivo tanto como meio de se prevenir a neoplasia do colo do útero, quanto como meio de diagnóstico precoce. Este fato pode ser explicado pela ampla divulgação do exame por meio das campanhas governamentais e mídias. Um estudo recente mostrou que mesmo as mulheres que possuem baixo nível de conhecimento sobre a neoplasia do colo do útero e, também, o não reconhecimento do HPV como fator de risco necessário, sabem sobre os métodos de rastreamento das lesões, fato que foi atribuído às informações provenientes dos meios de comunicação ${ }^{23}$.

Entre os motivos que levaram as mulheres a realizar o exame preventivo, nota-se que as que o fizeram há mais de dois anos apontaram que toda mulher entre 25 a 64 anos deve fazê-lo, o que parece contraditório, pois quando questionadas sobre a faixa etária indicada para se realizar o exame, a maioria assinalou outras faixas etárias. Já as que fizeram o exame preventivo há menos de dois anos, o motivo apontado foi considera importante fazêlo, um motivo que denota pouco conhecimento e sugere que medidas que proponham o conhecimento sejam reafirmadas. A principal razão apontada pelas mulheres para realizarem o exame reside na rotina ginecológica, motivo esse que se fez presente em apenas algumas respostas das entrevistadas ${ }^{24}$.

Evidencia-se também no presente estudo que a maioria das entrevistadas prefere receber informações sobre saúde a partir de palestras. Estudo anterior verificou que as mulheres, que são o foco das ações de rastreio da neoplasia do colo do útero, preferem receber informações por meio de panfletos e palestras ${ }^{25}$. Em um estudo sistemático, comprovou-se que qualquer intervenção educacional pode melhorar a adesão das mulheres aos programas de rastreamento, bem como diminuir as barreiras encontradas por elas ${ }^{26,27}$. De qualquer forma, pode-se também inferir que o desconhecimento e a falta de convivência com outros meios para se obter informações explica a opção da maioria delas por palestras, abrindo, portanto, uma nova possibilidade a ser explorada em outros estudos, visto que a educação oferece subsídios para se adotar novos hábitos e comportamentos condicionantes de saúde?

Por trabalharem na mesma IES e exercerem a mesma atividade laboral, essas mulheres estabelecem vínculos de amizade entre si e convívio com os membros universitários. Isto termina por garantir um ambiente oportuno para o desenvolvimento de medidas educativas de saúde levando ao empoderamento dessas pessoas. Tal conclusão alinha-se ao afirmado em um estudo de revisão sistemática acerca das Universidades Promotoras da Saúde ${ }^{12}$. Este apontou que a participação dos membros universitários em um processo de efetiva implementação de ações em saúde, precedido de planejamento e avaliação, é uma contribuição extremamente valiosa para a promoção da saúde.

Os resultados obtidos apontam que as mulheres analisadas têm baixo conhecimento sobre a adesão ao exame preventivo e, muitas vezes, realizam o procedimento sem a devida compreensão de sua importância. Para aquelas que nunca realizaram o exame, as características se assemelham muito às classicamente descritas em outros estudos, ou seja, baixa escolaridade e baixa renda.

\section{CONCLUSÃO}

A consciência de que o empoderamento em saúde contribui para a tomada de decisões individuais que podem salvar vidas impulsionou esse estudo, inserindo-o no paradigma da promoção da saúde.

A promoção da saúde é aqui entendida numa perspectiva ampliada, isto é, vincula-se tanto aos comportamentos individuais quanto ao comprometimento com a justiça social e aos direitos 
humanos. Nesse aspecto, renda e escolaridade são pilares estratégicos.

Diante do exposto, fica evidente a importância das ações de educação em saúde, notadamente no âmbito de uma instituição agraciada com o título de Universidade Promotora da Saúde.

Acredita-se que os dados aqui apresentados possam ser relevantes para o delineamento futuro de estratégias direcionados ao empoderamento de mulheres sobre a prevenção da neoplasia do colo do útero, especialmente no que tange aos espaços de integração entre universidade e comunidade.

\section{REFERÊNCIAS}

1. Lopez MS, Baker ES, Maza M, Fontes-Cintra G, Lopez A, Carvajal JM, et al. Cervical cancer prevention and treatment in Latin America. J Surg Oncol. [Internet] 2017 Apr; [citado 2018 Jul 27]. 115(5):615-8. Disponível em: $<$ https://onlinelibrary.wiley.com/doi/abs/10.1002/ jso.24544>. DOI: https://doi.org/10.1002/ jso. 24544

2. Instituto Nacional de Câncer Jose Alencar Gomes da Silva (INCA). Estimativa 2018: incidência de câncer no Brasil - síntese de resultados e comentários. [Internet]. Rio de Janeiro: INCA, 2018. [citado 2018 Jul 27]. Disponível em: <http:// www1.inca.gov.br/estimativa/2018/sintese-deresultados-comentarios.asp $>$.

3. Instituto Nacional de Câncer Jose Alencar Gomes da Silva (INCA). Perguntas mais frequentes: HPV câncer do colo do útero. [Internet]. Rio de Janeiro: INCA, 2018. [citado 2018 Jul 25]. Disponível em: $<$ http://www2.inca.gov.br/wps/wcm/connect/ tiposdecancer/site/home/colo_utero/hpv-cancerperguntas-mais-frequentes $>$.

4. Instituto Nacional de Câncer Jose Alencar Gomes da Silva (INCA). Ações e Programas no Brasil: controle do câncer do colo do útero. [Internet]. Rio de Janeiro: INCA; 2018. [citado 2018 Jul 27]. Disponível em: < http://www2.inca.gov.br/wps/ wcm/connect/acoes_programas/site/home/nobrasil/ programa_nacional_controle_cancer_colo_utero/ deteccao_precoce $>$.
5. Instituto Nacional de Câncer Jose Alencar Gomes da Silva (INCA). Parâmetros técnicos para o rastreamento do câncer do colo do útero. [Internet]. Rio de Janeiro: INCA; 2019. [citado 2019 Jul 27]. Disponível em: <https://www.inca.gov. br/sites/ufu.sti.inca.local/files//media/document// parametros_tecnicos_colo_do_utero_2019.pdf > .

6. Girianelli VR, Gamarra CJ, Silva GA. Os grandes contrastes na mortalidade por câncer do colo uterino e de mama no Brasil. Rev. Saúde Pública [Internet]. 2014 Jun. [citado 2019 Sep 02]; 48(3):459-67. Disponível em: DOI: http://dx.doi. org/10.1590/S0034-8910.2014048005214.

7. Instituto Nacional de Câncer Jose Alencar Gomes da Silva (INCA). Coordenação de Prevenção e Vigilância. Divisão de Detecção Precoce e Apoio à Organização de Rede. Diretrizes brasileiras para o rastreamento do câncer do colo do útero. 2. ed. rev. Rio de Janeiro: INCA; 2016.

8. Aguilar RP, Soares DA. Barreiras à realização do exame Papanicolau: perspectivas de usuárias e profissionais da Estratégia de Saúde da Família da cidade de Vitória da Conquista-BA. Physis. 2015; 25(2):359-79.

9. Souza G, Alves OS. Estratégias educativas para prevenção e redução da morbimortalidade do câncer do colo uterino. SaudPesq. 2015; 8(2):31726.

10. Chiesa AM, Zoboli ELCP, Granja GF. Atenção à saúde na perspectiva da equidade. Pelicioni MCF, Mialhe FL, organizadores. Educação e promoção da saúde: teoria e prática. Rio de Janeiro: Santos; 2019. p. Xxx-xxx.

11. Luszczynska A, Durawa AB, Scholz U, Knoll N. Empowerment beliefs and intention to uptake cervical cancer screening: three psychosocial mediating mechanisms. J Women \& Health. [Internet]. 2012 [citado 2018 Jul 27]; 52(2): 162-81. Disponível em: <https:/kops.unikonstanz.de/bitstream/handle/123456789/20974/ Scholz_209746.pdf;sequence $=2>$. DOI: https:// doi.org/10.1080/03630242.2012.656187.

12. Suárez-Reyes M, Van-den-Broucke S. Implementing 
the Health Promoting University approach in culturally different contexts: a systematic review. IUHPE - Global Health Promotion. [Internet]. 2016 [citado 2018 Jul 27]; 23(Supp. 1):46-6. Disponível em: < https:/journals.sagepub.com/doi/ full/10.1177/1757975915623933 > DOI: https:// doi.org/10.1177/1757975915623933.

13. Maniva SJCF, Carvalho ZMF, Gomes RKG, Carvalho REFL, Ximenes LB, Freitas CHA. Tecnologias educativas para educação em saúde no acidente vascular cerebral: revisão integrativa. Rev. Bras. Enferm. [Internet]. 2018 [citado 2019 Set 02]; 71(Suppl 4):1724-31. Disponível em: < http:// www.scielo.br/pdf/reben/v71s4/pt_0034-7167reben-71-s4-1724.pdf> . DOI: http://dx.doi. org/10.1590/0034-7167-2016-0693.

14. Ribeiro CM, Dias MBKneipp, Sole Pla MA, Correa FM, Russomano FB, Tomazelli JG. Parâmetros para a programação de procedimentos da linha de cuidado do câncer do colo do útero no Brasil. Cad. Saúde Pública [Internet]. 2019 [citado 2019 Set 02]; 35(6): e00183118. Disponível em: http://www.scielo.br/scielo. php?script $=$ sci_arttext\&pid $=$ S0102311X2019000705008\&lng=en. DOI: http://dx.doi. org/10.1590/0102-311x00183118.

15. Teixeira JC, Maestri CA, Machado HC, Zeferino LC, Carvalho NS. Cervical Cancer registered in two developed regions from Brazil: upper limit of reachable results from opportunistic screening. Rev. Bras. Ginecol. Obstet. [Internet]. 2018 June [citado 2018 Oct 17]; 40(6):34753. Disponível em: < http://www.scielo.br/ scielo.php?script $=$ sci_arttext\&pid $=S 0100$ 72032018000600347\&lng=en > . DOI: http:// dx.doi.org/10.1055/s-0038-1660841.

16. Silva RCG, Silva ACO, Peres AL, Oliveira SR. Perfil de mulheres com câncer de colo do útero atendidas para tratamento em centro de oncologia. Rev. Bras. Saude Mater. Infant. [Internet]. 2018 Dec [citado 2019 Set 01]; 18(4):695-702. Disponível em: <http://www.scielo. $\mathrm{br} / \mathrm{scielo}$.php?script $=$ sci_arttext $\&$ pid $=S 1519$ 38292018000400695\&lng =en > . DOI: http:// dx.doi.org/10.1590/1806-93042018000400002.
17. Hamoonga TE, Likwa RN, Musonda P, Michelo C. Higher educational attainment associated with reduced likelihood of abnormal cervical lesions among Zambian women - a cross sectional study. BMC Cancer. [Internet]. 2017; [citado 2019 Set 01]; 17(1):1-12. Disponível em: < https:/bmccancer. biomedcentral.com/articles/10.1186/s12885-0173680-z> . DOI: https://doi.org/10.1186/s12885-0173680-z.

18. Broberg G, Wang J, Östberg AL, Adolfsson A, Nemes S, et al. Socio-economic and demographic determinants affecting participation in the Swedish cervical screening program: A population-based case-control study. PLOS ONE. [Internet]. 2018 [citado 2019 Set 01]; 13(1): e0190171. Disponível em: < Socio-economic and demographic determinants affecting participation in the Swedish cervical screening program: A population-based case-control study >. DOI: https://doi.org/10.1371/ journal.pone. 0190171

19. Costa PF, Cerqueira-Santos E. Fatores associados ao uso de preservativo e relações com prostitutas entre caminhoneiros do Brasil. Psic., Saúde \& Doenças. [Internet]. 2018 Dez [citado 2019 Set 01]; 19(3):617-27. Disponível em: < http://www.scielo. mec.pt/scielo.php?script $=$ sci_arttext\&pid $=$ S164500862018000300012\&lng = pt > . DOI: http:// dx.doi.org/10.15309/18psd1912.

20. Nascimento EGC, Cavalcanti MAF, Alchieri JC. Adesão ao uso da camisinha: a realidade comportamental no interior do Nordeste do Brasil. Rev. Salud pública [Internet]. $2017 \mathrm{Fev}$ [citado 2018 Out 16]; 19(1):39-44. Disponível em: < https://www.scielosp.org/article/rsap/2017. v19n1/39-44/pt/> . DOI https://doi.org/10.15446/ rsap.v19n1.44544.

21. Conde C, Lemos TM, Ferreira ML. Características sociodemográficas, individuales y de programación de las mujeres con cáncer cervical. Enferm. glob. [Internet] 2018 [citado 2018 Out 16]; 17(1):34880. Disponível em: < http://revistas.um.es/eglobal/ article/view/301041>.

22. Larasati L, Afiyanti Y, Rahmab H, Milanti A. Women's 
knowledge, beliefs, and behaviors toward the prevention of human papillomavirus transmission.

Recebido em: 30/07/2019

Enfermería Clínica. [Internet] 2018 [citado 2018

Out 16]; 28(Suppl 1):191-4. Disponível em:

Aceito em: 11/09/2019

$<$ https://www.sciencedirect.com/science/article/

pii/S1130862118300652>. DOI http://dx.doi.

org/10.1016/s1130-8621(18)30065-2

23. Mruts K, Gebremariam T. Conhecimento e percepção em relação ao câncer cervical entre estudantes universitarias Debre Berhan. J Pacífico Asiático de Preven Câncer. [Internet] 2018 [citado 2018 Out 16]; 19(7):1771-7. Disponível em: https://10.22034/APJCP.2018.19.7.1771.

24. Navarro C, Fonseca AJ, Sibajev A, Souza CIA, Araújo DS, Teles DAF, et al. Cervical cancer screening coverage in a high-incidence region. Rev. Saúde Pública. [Internet] 2015 [citado 2018 Out 16]; 49:17. Disponível em: < https://www.ncbi.nlm. nih.gov/pmc/articles/PMC4386559/> . DOI: http:// dx.doi.org/10.1590/S0034-8910.2015049005554.

25. Bilotti CC, Nepomuceno LD, Altizani GM, Macuch RS, Lucena TFR, Bortolozzi F, et al. M-Health no controle do câncer de colo do útero: pré-requisitos para o desenvolvimento de um aplicativo para smartphones. Reciis: Rev Eletron Comun Inf Inov Saúde. [Internet] 2017 [citado 2018 Out 16]; 11(2):1-18. Disponível em: < https://www.arca. fiocruz.br/bitstream/icict/19705/3/ve_Bilotti_ Carolina_Correia_etal_2017.pdf $>$.

26. Naz MSG, Kariman N, Ebadi A, Ozgoli G, Ghasemi V, Fakari FR. Educational interventions for Cervical Cancer Screening Behavior of Women: a systematic review. Asian Pac J Cancer Prev. [Internet] 2018 [citado 2018 Out 16]; 19(4):875-84. DOI: 10.22034/ APJCP.2018.19.4.875.

27. Pereira JD, Lemos MS. Preditores motivacionais de adesão à prevenção do câncer do colo do útero em estudantes universitárias. Estud. psicol. (Campinas). [Internet] 2019 [citado 2019 Set 04]; 36:e170073. Disponível em: <http://www.scielo. br/scielo.php?script $=$ sci_arttext\&pid $=$ S0103166X2019000100902 >. DOI https://dx.doi. org/10.1590/1982-0275201936e170073. 